\title{
SOFA score is superior to APACHE-II score in predicting the prognosis of critically ill patients with acute renal injury undergoing continuous renal replacement therapy
}

Hai Wang

Xi'an Jiaotong University

Yu Shi

Xi'an Jiaotong University

Zheng-hai Bai

Xi'an Jiaotong University

Jun-hua LV

Xi'an Jiaotong University

Jiang-li Sun

Xi'an Jiaotong University

Hong-hong Pei

Xi'an Jiaotong University

Zhengliang Zhang ( $\square$ zhangzljz@163.com )

Xi'an Jiaotong University

Research article

Keywords: SOFA score, APACHE-II score, acute renal injury, continuous renal replacement therapy.

Posted Date: July 3rd, 2019

DOI: https://doi.org/10.21203/rs.2.10965/v1

License: (c) (1) This work is licensed under a Creative Commons Attribution 4.0 International License.

Read Full License

Version of Record: A version of this preprint was published at Renal Failure on January 1st, 2020. See the published version at https://doi.org/10.1080/0886022X.2020.1788581. 


\section{Abstract}

Background: Acute renal injury (AKI) is the most common cause of organ failure in multiple organ dysfunction syndrome and is associated with higher mortality, especially for patients requiring continuous renal replacement therapy (CRRT). At present, no effective approaches are in place to predict the prognosis of patients with AKI undergoing CRRT. Methods: A retrospective cohort study was carried to determine whether sequential organ failure assessment (SOFA) score may be a more valuable prognostic indicator than acute physiology and chronic health evaluation II (APACHE-II) score in patients with AKI undergoing CRRT. The multivariate analysis, sensitivity analysis, receiver operating characteristics (ROC) curve and decision curve analysis (DCA) were performed to determine the predictive value of SOFA and APACHE-II scores on 28- and 90-day mortality. Results: From January 2009 to September 2016, a total of 1128 cases were included. Multivariate logistic regression analysis showed that both APACHE-II and SOFA scores were associated with 28- and 90-day mortality of patients with AKI undergoing CRRT. The adjusted odds ratios (ORs) for SOFA and APACHE-II scores associated with 28-day mortality were 1.38 $(1.27,1.51)$ and $1.04(1.01,1.07)$, respectively, and the adjusted ORs for SOFA and APACHE-II scores associated with 90-day mortality were $1.40(1.28,1.52)$ and $1.04(1.01,1.07)$, respectively. decision curve analysis and receiver operating characteristics analyses showed that SOFA had a higher prediction accuracy than APACHE-II for 28- and 90-day mortality. The AUCs of SOFA and APACHE-II for 28-day mortality were $0.70(0.67,0.73)$ and $0.62(0.58,0.65)$, and for 90 -day mortality, the AUCs were $0.71(0.68$, $0.78)$ and $0.62(0.58,0.65)$, respectively. Conclusion: SOFA score revealed higher accuracy than APACHE-II score in predicting the prognosis of critically ill patients with AKI undergoing CRRT.

\section{Background}

Multiple organ dysfunction syndrome (MODS) is the main cause of death in patients in the intensive care unit (ICU) ${ }^{[1]}$. Acute renal injury (AKI) is the most common organ failure syndrome in MODS ${ }^{[2-4]}$ and the one with higher mortality, especially for patients requiring continuous renal replacement therapy (CRRT) $[5,6]$. Research has shown that the prognosis of these patients is related to the number of failed organs and the degree of organ failure ${ }^{[7,8]}$. Evaluation of organ function in critically ill patients is helpful in estimating their prognosis ${ }^{[9]}$. Therefore, a variety of scoring systems such as acute physiology and chronic health evaluation (APACHE), Marshall method (MOF) and MOD score [10,11], among others, have emerged, with APACHE-II being widely used ${ }^{[12]}$. However, this score is not able to evaluate changes in organ function. The sequential organ failure assessment (SOFA) score was developed by an international group of experts to describe multiple organ dysfunction using a limited number of routinely measured variables ${ }^{[13]}$. The SOFA score has been widely used in the diagnosis of sepsis and in identifying patients with sepsis and organ dysfunction or determining individual treatment strategies or outcomes of sepsis patients ${ }^{[14]}$.Similarly to the APACHE-II score, SOFA has been used to assess disease severity and predict prognosis of patients with cancer ${ }^{[15]}$, acute pancreatitis ${ }^{[16]}$, acute liver failure ${ }^{[17]}$ and acute respiratory distress syndrome (ARDS) ${ }^{[18]}$. However, it is not clear whether SOFA score can be used to assess the 
prognosis of patients with AKI undergoing CRRT. Therefore, we hypothesized that SOFA score may be a valuable prognostic indicator for patients with AKI undergoing CRRT.

\section{Methods}

\section{Methods:}

Study design: A retrospective cohort study.

Objective: We aimed to detect the value of SOFA score in predicting the prognosis of patients with AKI undergoing CRRT.

Data source: The data of our study was derived from the Dryad database which was a curated resource that makes the data underlying scientific publications discoverable, freely reusable, and citable. The data in our study was provided by Seung Hyeok Han in Dryad database ${ }^{[19,20]}$ and the study was carried out at Yonsei University Health System Severance Hospital and National Health Insurance Service Medical Center Ilsan hospital.

Inclusion criteria: Patients with (1) Acute Kidney Injury Network (AKIN) stage 2 and (2) treated with CRRT.

Exclusion criteria: (1) age $\leq 18$ years ; (2) previous chronic kidney disease (CKD), dialysis or CRRT; (3) pregnancy or lactation; (4) postrenal obstruction; (5) kidney transplantation and (6) missing SOFA and APACHE-II score values.

Participants: From January 2009 to September 2016, 2391 patients were treated with CRRT in the ICU at Yonsei University Health System Severance Hospital and National Health Insurance Service Medical Center Ilsan hospital. Among them, 2110 patients met the inclusion criteria and 982 met the exclusion criteria: age $\leq 18$ years $(n=42)$, previous CKD, dialysis or CRRT $(n=585)$, pregnancy $(n=12)$, postrenal obstruction $(n=263)$, kidney transplantation $(n=64)$ and missing score values $(n=16)$. Finally, a total of 1128 cases which met the inclusion and exclusion criteria were included in our study.

Clinical and biochemical data collection: the collected variables included demographic, complications, biochemical laboratory results and disease severity index at Oh of CRRT. The detailed variables were as follows: sex, body mass index (BMI), mean arterial pressure (MAP), CRRT cause, comorbidities, hemoglobin (HB), white blood cell (WBC) count, serum creatinine (Cr), phosphate, albumin (Alb), bicarbonate ( $\mathrm{HCO}-)$, potassium $(\mathrm{K}+)$, blood urea nitrogen (BUN), C-reactive protein (CRP) and glomerular filtration rate (GFR),SOFA score, APACHE-II score and Charlson comorbidity index (CCI).

The outcome indicators: the outcome indicators included 28-day mortality and 90-day mortality.

CRRT protocol: CRRT initiation was decided by nephrologists based on AKI development in ICU patients. Indications for CRRT included metabolic acidosis, intractable hyperkalemia or uncontrolled volume 
overload. The CRRT protocol consisted of continuous veno-venous hemofiltration through the internal jugular, subclavian or femoral veins. Initial CRRT blood flow rate was $100 \mathrm{~mL} / \mathrm{min}$ and up to $150 \mathrm{~mL} / \mathrm{min}$. The summed targeted dialysis and replacement dose was targeted all patients.

Statistical analysis: (1) Statistical description: mean \pm standard deviation $(x \pm s)$ was used for continuous variables of baseline data, and absolute values and percentages were used for categorical variables. (2) Univariate analysis was used to detect the risk associated with 28- and 90-day mortality. (3) In multivariate analysis, variables possibly affecting patients' prognosis were adjusted to determine the effect of SOFA or APACHE-II scores on 28- and 90-day mortality. (4) Sensitivity analysis was performed by considering sepsis and non-sepsis to further verify the effect of SOFA or APACHE-II scores on 28- and 90day mortality. (5) Receiver operating characteristics (ROC) curve and decision curve analysis (DCA) were used to determine the effect of SOFA and APACHE-II scores on 28- and 90-day mortality. $\mathrm{P}<0.05$ was considered for statistical significance. All statistical analyses were performed with EmpowerStats (version numbers: 2018-12-22, Copyright 2009 X\&Y Solutions, Inc.) and R software.

\section{Results}

\section{Baseline characteristics}

The clinical characteristics and laboratory findings for all patients are shown in Table 1. A total of 1128 cases met the inclusion and exclusion criteria and were included in the study. The mean age was $63.12 \pm$ 14.46 years, 695 (61.2\%) patients were male, and the mean BMl and MAP were $23.80 \pm 4.59 \mathrm{~kg} / \mathrm{m} 2$ and $77.43 \pm 14.62 \mathrm{mmHg}$, respectively. The numbers of patients with myocardial infarction, congestive heart failure, cerebrovascular disease, peripheral vascular disease, dementia, diabetes, hypertension and COPD (chronic obstructive pulmonary disease) were 110 (9.75\%), 181 (16.05\%), 114 (10.14\%), 44 (3.90\%), 42 (3.72\%), 395 (35.05\%), 591 (52.39\%) and 79 (7.00\%), respectively. The number of patients with mechanical ventilation at the beginning of CRRT was 888 (78.79\%). The mean SOFA and APACHE-II scores were $12.12 \pm 3.55$ and $27.30 \pm 7.98$, respectively (see table 1 ).

\section{Univariate analysis results}

In univariate analysis, $\mathrm{BMI}, \mathrm{MAP}$, hypertension, $\mathrm{Hb}, \mathrm{Cr}$, and $\mathrm{Alb}$ were the protective factors, and mechanical ventilation, phosphate, GFR, APACHE-II score, SOFA score, CCI score, CRRT cause and AKI cause were the risk factors for 28-day mortality. On the other hand, BMI, MAP, diabetes mellitus, hypertension, $\mathrm{Hb}, \mathrm{Cr}$ and Alb were the protective factors, and mechanical ventilation, phosphate, BUN, APACHE-II score, SOFA score, $\mathrm{CCl}$ score, CRRT cause and AKI cause were the risk factors for 90-day mortality. (see table 2)

\section{Multivariate logistic regression analysis results}

In multivariate logistic regression analysis, we found that SOFA score and APACHE II score were all associated with the 28-day mortality and 90 -day mortality of patients with AKI treated CRRT. The adjusted ORs of SOFA score were respectively $1.38(1.27,1.51)$ and $1.40(1.28,1.52)$ for the 28 -day mortality and 
90-day mortality. The adjusted ORs of APACHE II score were respectively $1.04(1.01,1.07)$ and 1.04 (1.01, 1.07) for the 28-day mortality and 90-day mortality.(see table3)

\section{Multivariate subgroup regression analysis results based on AKI causes}

In multivariate subgroup regression analysis, possible confounding factors associated with 28- and 90day mortality in patients with AKI undergoing CRRT were also adjusted. Results showed that SOFA score was a risk factor for 28- and 90-day mortality and that APACHE-II score was not a risk factor of 28- and 90-day mortality both in sepsis and non-sepsis patients. In sepsis patients, the adjusted ORs of SOFA and APACHE-II for 28-day mortality were $1.38(1.23,1.55)$ and $1.03(0.99,1.07)$, and the adjusted ORs of SOFA and APACHE-II for 90-day mortality were $1.41(1.26,1.58)$ and $1.02(0.98,1.06)$, respectively. In non-sepsis patients, the adjusted ORs of SOFA and APACHE-II for 28-day mortality were $1.66(1.26,2.19)$ and 1.04 $(0.97,1.12)$, and the adjusted ORs of SOFA and APACHE-II for 90-day mortality were $1.36(1.06,1.74)$ and $1.03(0.95,1.12)$, respectively. (see table4)

\section{DCA and ROC results}

Both DCA and ROC analyses showed that SOFA score had a higher prediction accuracy than APACHE-II score for 28- and 90-day mortality in patients with AKI undergoing CRRT. The AUCs of SOFA and APACHEII for 28-day mortality were $0.70(0.67,0.73)$ and $0.62(0.58,0.65)$, respectively. The AUCs of SOFA and APACHE-II for 90 -day mortality were $0.71(0.68,0.78)$ and $0.62(0.58,0.65)$, respectively. In sepsis patients, the AUCs of SOFA and APACHE-II for 28-day mortality were $0.69(0.66,0.73)$ and $0.60(0.56$, 0.64), respectively, and the AUCs of SOFA and APACHE-II for 90 -day mortality were $0.72(0.66,0.77)$ and $0.65(0.59,0.71)$, respectively. In non-sepsis patients, the AUCs of SOFA and APACHE-II for 28-day mortality were $0.71(0.67,0.75)$ and $0.60(0.55,0.64)$, respectively, and the AUCs of SOFA and APACHE-II for 90 -day mortality were $0.73(0.67,0.78)$ and $0.65(0.59,0.72)$, respectively. (see figure 1 , figure 2 ,figure3 and figure4)

\section{Discussion}

In this study, SOFA and APACHE-II scores were found to be associated with 28- and 90-day mortality in patients with AKI undergoing CRRT. In the subgroup analysis, only SOFA score was found to be associated with 28- and 90-day mortality in patients with AKI undergoing CRRT. Both DCA and ROC analyses showed that SOFA score had a higher prediction accuracy than APACHE-II score for 28- and 90day mortality in patients with AKI undergoing CRRT.

APACHE-II score is one of the most commonly used predictive scoring systems for critically ill patients and has been widely used in predicting their prognosis ${ }^{[21-23]}$. However, several studies reported conflicting predictive accuracy results associated with APACHE-II score in critically ill patients. A prospectively defined analysis of a registry-based validation cohort including 3008 patients showed that the AUCs of APACHE-II for ICU mortality and hospital mortality were $0.81(0.79,0.82)$ and $0.77(0.76-$ 0.79), respectively [24]. A prospective cohort study of 522 patients admitted to the ICU with solid tumors 
showed that APACHE-II score had a poor predictive value in hospital mortality of these patients, with an AUC of $0.62(0.54,0.70){ }^{[25]}$. A retrospective study including 104 cases showed that APACHE-II score was a poor predictor of mortality in patients with epilepticus status in the ICU, with an AUC of $0.58(0.45,0.72)$ [26].

The SOFA score, previously known as the sepsis-related organ failure assessment score ${ }^{[27-29]}$, is used to assess organ function failure probability. The score is based on six different aspects related with respiratory, cardiovascular, hepatic, coagulation, renal and neurological systems. Recent studies found that SOFA was not only a useful tool for condition evaluation and prognosis prediction in sepsis patients but also a widely used tool in prognosis and condition assessment in other critically ill patients. Bodin Khwannimit conducted a research including 1.589 sepsis patients and found that SOFA score was a good predictor of 30-day and in-hospital mortality among sepsis patients in ICU, with an AUC of 0.88 [30]. In Ming-Chin Yu's study, the authors found that SOFA score was reliable in predicting mortality in acute severe pancreatitis, with an AUC of $0.76^{[31]}$. Another retrospective cohort study including 149 patients with hematological malignancies showed that SOFA score was a suitable prognostic indicator for ICU mortality in hematological malignancies patients ${ }^{[32]}$.

In this study, we found that APACHE-II score was a poor predictor for 28-and 90-day mortality in patients with AKI undergoing CRRT, but SOFA score was a more valuable predictor for both parameters in this patient population. A possible reason for this is the fact that acute renal failure is one of the most common types of organ failure in critically ill patients ${ }^{[2-4]}$. Additionally, patients with acute renal failure requiring CRRT frequently display multiple organ failure. Although SOFA score can be used to evaluate organ failure, APACHE-II score has a poor value in evaluating this parameter. Therefore, SOFA score has higher accuracy and value for prognosis evaluation in critically ill patients with AKI undergoing CRRT.

SOFA score is used to evaluate the prognosis of critically ill patients with AKI undergoing CRRT as it displays the following advantages compared with APACHE-II score: 1) SOFA score has relatively fewer items, being more convenient for clinical use ${ }^{[33]}$; 2) SOFA score is more suitable for prognosis evaluation

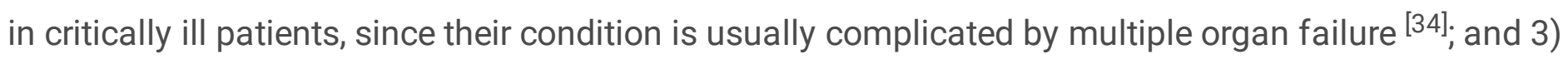
SOFA score is suitable for organ failure evaluation ${ }^{[13]}$.

Strength of the study: 1) This study renewed our knowledge that SOFA score has high accuracy in evaluating the prognosis of critically ill patients with AKI undergoing CRRT; 2) its results are more reliable than those of previous studies as a higher number of possible confounding factors were controlled.

Limitations of the study: The study only included patients with AKI undergoing CRRT, so the applicability of its conclusions is limited.

Conclusions: SOFA score has higher accuracy than APACHE-II score in predicting the prognosis of critically ill patients with AKI undergoing CRRT. 


\section{Abbreviations}

CRRT=continuous renal replacement therapy; $A K I=$ acute renal injury; SOFA= sequential organ failure assessment; APCHE II=acute physiology and chronic health evaluation Il; ROC=receiver operating characteristics curve; $\mathrm{DCA}=$ decision curve analysis; MODS= multiple organ dysfunction syndrome; $I C U=$ intensive care unit; $M O F=$ Marshall method; $A K I N=$ acute kidney injury network; $C K D=$ chronic kidney disease; $\mathrm{BMI}=$ body mass index; $\mathrm{MAP}=$ mean arterial pressure; $\mathrm{HB}=$ hemoglobin; $\mathrm{WBC}=$ white blood cell; $\mathrm{Cr}=$ serum creatinine; $\mathrm{Alb}=$ albumin; $\mathrm{HCO}-$ = bicarbonate; $\mathrm{K}+$ = potassium; $\mathrm{BUN}=$ blood urea nitrogen; $\mathrm{CRP}=\mathrm{C}$-reactive protein; $\mathrm{GFR}=$ glomerular filtration rate; $\mathrm{CCl}=$ Charlson comorbidity index; $\mathrm{COPD}=$ chronic obstructive pulmonary disease.

\section{Declarations}

Ethics approval and consent to participate: New ethics approval and consent to participate were not applicable, because the original author had obtained the ethical approval when conducting this study and our study was retrospective study of data reuse.

Consent to publish: The consent to publish was obtained from all authors.

Availability of data and materials: please see the website https://datadryad.org//resource/doi:10.5061/dryad.6v0j9.

Competing interests: The authors have no conflicts of interest to disclose.

Funding: Not applicable.

Authors' contributions: Hai Wang and Yu Shi wrote the manuscript, Jun hua Lv, Jiang-li Sun and Zhenghai Bai finished the statistical analysis, Hong-hong Pei and Zheng liang Zhang were responsible for research design, process guidance and checking and correction.

Acknowledgements: Thank for Seung Hyeok Han providing the data in the dryad database.

\section{References}

1. F H, D R, M P, et al. Prognostic Value of Tissue Oxygen Saturation Using a Vascular Occlusion Test in Patients in the Early Phase of Multiorgan Dysfunction Syndrome. Shock (Augusta, Ga.). 2018; undefined(undefined): undefined.

2. S S, AK P, B P, et al. Acute renal failure in the ICU setting: A prospective observational study. Medical journal, Armed Forces India. 2016;72(3):236-241.

3. JT K, O B. Dosing of antibiotics in critically ill patients undergoing renal replacement therapy. Current pharmaceutical biotechnology. 2011;12(12):2015-2019. 
4. MB I, EI N. [Hybrid technologies of renal replacement therapy in the treatment of multiple organ failure in cardiosurgical patients]. Anesteziologiia i reanimatologiia. 2006;undefined(3):67-70.

5. M M, M K, C W, et al. Regional citrate versus systemic heparin anticoagulation for continuous renal replacement therapy in critically ill patients with acute kidney injury $(\mathrm{RICH})$ trial: study protocol for a multicentre, randomised controlled trial. BMJ open. 2019;9(1):e024411.

6. C R, Z R. Pediatric continuous renal replacement: 20 years later. Intensive care medicine. 2015;41(6):985-993.

7. JM S, E C, K B. Contemporary Patterns of Multiple Organ Dysfunction in Trauma. Shock (Augusta, Ga.). 2017;47(4):429-435.

8. KX, C G, L S, P Y, X L, L X. Prognostic value of different scoring models in patients with multiple organ dysfunction syndrome associated with acute COPD exacerbation. Journal of thoracic disease. 2015;7(3):329-336.

9. Maccariello E, Rocha E, Valente $\mathrm{C}$, et al. Effects of early changes in organ dysfunctions on the outcomes of critically ill patients in need of renal replacement therapy. Clinics (Sao Paulo). Jun 2008;63(3):343-350.

10. L M, E F, M K, O T. Predictive ability of the ISS, NISS, and APACHE II score for SIRS and sepsis in polytrauma patients. European journal of trauma and emergency surgery : official publication of the European Trauma Society. 2012;38(6):665-671.

11. Huang W, Qin S, Sun Y, et al. [Establishment of multiple organ dysfunction syndrome early warning score in patients with severe trauma and its clinical significance: a multicenter study]. Zhonghua Wei Zhong Bing Ji Jiu Yi Xue. Jan 2018;30(1):41-46.

12. M B, SS E, N M, V R, N M. Acute Physiology and Chronic Health Evaluation II score for the assessment of mortality prediction in the intensive care unit: a single-centre study from Iran. Nursing in critical care. 2019; undefined(undefined):undefined.

13. Singer M, Deutschman CS, Seymour CW, et al. The Third International Consensus Definitions for Sepsis and Septic Shock (Sepsis-3). Jama. Feb 23 2016;315(8):801-810.

14. Fernando SM, Rochwerg B, Seely AJE. Clinical implications of the Third International Consensus Definitions for Sepsis and Septic Shock (Sepsis-3). Cmaj. Sep 10 2018;190(36):E1058-e1059.

15. H K, M Y, R G, et al. Predictors of Survival in Patients with Advanced Gastrointestinal Malignancies Admitted to the Intensive Care Unit. The oncologist. 2018; undefined(undefined):undefined.

16. YS T, HY F, IM K, YS L, SF H, MC Y. Serial evaluation of the SOFA score is reliable for predicting mortality in acute severe pancreatitis. Medicine. 2018;97(7):e9654.

17. TL G, RC F, LL V, et al. Low vitamin $D$ at ICU admission is associated with cancer, infections, acute respiratory insufficiency, and liver failure. Nutrition (Burbank, Los Angeles County, Calif.). 2019;60(undefined):235-240.

18. SH L, MJ H, SW L, et al. Outcomes of severe H1N1 pneumoniae: A retrospective study at intensive care units. Journal of the Formosan Medical Association = Taiwan yi zhi. 2019; undefined(undefined): undefined. 
19. Jung SJ, Kwon J, Park S, et al. Data from: Phosphate is a potential biomarker of disease severity and predicts adverse outcomes in acute kidney injury patients undergoing continuous renal replacement therapy: Dryad Digital Repository; 2018.

20. Jung SY, Kwon J, Park S, et al. Phosphate is a potential biomarker of disease severity and predicts adverse outcomes in acute kidney injury patients undergoing continuous renal replacement therapy. PLoS One. 2018;13(2):e0191290.

21. Delibegovic S, Markovic D, Hodzic S. APACHE II scoring system is superior in the prediction of the outcome in critically ill patients with perforative peritonitis. Med Arh. 2011;65(2):82-85.

22. Imaura M, Yokoyama H, Kohata $Y$, et al. Initial dosing regimen of vancomycin to achieve early therapeutic plasma concentration in critically ill patients with MRSA infection based on APACHE II score. Eur J Drug Metab Pharmacokinet. Jun 2016;41(3):211-218.

23. Nakhoda S, Zimrin AB, Baer MR, Law JY. Use of the APACHE II score to assess impact of therapeutic plasma exchange for critically ill patients with hypertriglyceride-induced pancreatitis. Transfus Apher Sci. Apr 2017;56(2):123-126.

24. Falcao ALE, Barros AGA, Bezerra AAM, et al. The prognostic accuracy evaluation of SAPS 3 , SOFA and APACHE II scores for mortality prediction in the surgical ICU: an external validation study and decision-making analysis. Ann Intensive Care. Jan 30 2019;9(1):18.

25. Martos-Benitez FD, Cordero-Escobar I, Soto-Garcia A, Betancourt-Plaza I, Gonzalez-Martinez I. APACHE II score for critically ill patients with a solid tumor: A reclassification study. Rev Esp Anestesiol Reanim. Oct 2018;65(8):447-455.

26. Cheng JY. Mortality prediction in status epilepticus with the APACHE II score. J Intensive Care Soc. Nov 2017;18(4):310-317.

27. W Z, Y Z, X F, M C, Y K. Systemic inflammatory response syndrome in Sepsis-3: a retrospective study. BMC infectious diseases. 2019;19(1):139.

28. M K, B S, S C, et al. Red-flag sepsis and SOFA identifies different patient population at risk of sepsisrelated deaths on the general ward. Medicine. 2018;97(49):e13238.

29. H F, M K, K K, S M. Performance of Quick Sequential (Sepsis Related) and Sequential (Sepsis Related) Organ Failure Assessment to Predict Mortality in Patients with Acute Pyelonephritis Associated with Upper Urinary Tract Calculi. The Journal of urology. 2018;199(6):1526-1533.

30. Khwannimit B, Bhurayanontachai R, Vattanavanit V. Comparison of the accuracy of three early warning scores with SOFA score for predicting mortality in adult sepsis and septic shock patients admitted to intensive care unit. Heart Lung. Mar 192019.

31. Tee YS, Fang HY, Kuo IM, Lin YS, Huang SF, Yu MC. Serial evaluation of the SOFA score is reliable for predicting mortality in acute severe pancreatitis. Medicine (Baltimore). Feb 2018;97(7):e9654.

32. Aamp D, Geerse DA, Bjp J, Winkens B, Schouten HC, Wnka VM. The prognostic value of a trend in modified SOFA score for patients with hematological malignancies in the intensive care unit. European Journal of Haematology. 2017. 
33. $\mathrm{KM} \mathrm{H}$. Combining sequential organ failure assessment (SOFA) score with acute physiology and chronic health evaluation (APACHE) II score to predict hospital mortality of critically ill patients. Anaesthesia and intensive care. 2007;35(4):515-521.

34. E E, AN C, MS B, et al. Risk factors for infection and evaluation of Sepsis-3 in patients with trauma. American journal of surgery. 2019; undefined(undefined):undefined.

\section{Tables}


Table1. The clinical characteristics of patients

\begin{tabular}{|c|c|}
\hline Patient Characteristics & Mean $\pm \mathrm{SD} / \mathrm{N}(\%)$ \\
\hline Age, year & $63.12 \pm 14.46$ \\
\hline $\operatorname{Sex}(M / F)$ & $695 / 433$ \\
\hline $\mathrm{BMI}, \mathrm{Kg} / \mathrm{m}^{2}$ & $23.80 \pm 4.59$ \\
\hline $\mathrm{MAP}, \mathrm{mmHg}$ & $77.43 \pm 14.62$ \\
\hline Myocardial infarction, n (\%) & $110(9.75 \%)$ \\
\hline Congestive heart failure, n (\%) & $181(16.05 \%)$ \\
\hline Cerebrovascular disease, n (\%) & $114(10.14 \%)$ \\
\hline peripheral vascular disease, $\mathrm{n}(\%)$ & $44(3.90 \%)$ \\
\hline Dementia, n (\%) & $42(3.72 \%)$ \\
\hline Diabetes, n (\%) & 395 (35.05\%) \\
\hline Hypertension, n (\%) & 591 (52.39\%) \\
\hline COPD, n (\%) & 79 (7.00\%) \\
\hline Mechanical ventilation, n (\%) & $888(78.79 \%)$ \\
\hline $\mathrm{K}^{+}, \mathrm{mmol} / \mathrm{L}$ & $4.70 \pm 1.10$ \\
\hline $\mathrm{HCO}^{-}, \mathrm{mmol} / \mathrm{L}$ & $16.91 \pm 5.73$ \\
\hline Phosphate, mmol/L & $5.75 \pm 2.43$ \\
\hline $\mathrm{WBC}, 10^{9} / \mathrm{L}$ & $14.12 \pm 13.20$ \\
\hline $\mathrm{Hb}, \mathrm{g} / \mathrm{L}$ & $9.63 \pm 2.23$ \\
\hline BUN, mg/dL & $55.85 \pm 29.98$ \\
\hline $\mathrm{Cr}, \mathrm{mg} / \mathrm{dL}$ & $2.73 \pm 1.62$ \\
\hline Alb, $g / L$ & $2.61 \pm 0.58$ \\
\hline $\mathrm{CRP}, \mathrm{mg} / \mathrm{L}$ & $110.26 \pm 107.96$ \\
\hline GFR, \% & $31.30 \pm 21.21$ \\
\hline APACHE II score & $27.30 \pm 7.98$ \\
\hline SOFA score & $12.12 \pm 3.55$ \\
\hline \multicolumn{2}{|l|}{ AKIN stage } \\
\hline 2 & $292(25.89 \%)$ \\
\hline 3 & $836(74.11 \%)$ \\
\hline CCI score & $3.16 \pm 2.24$ \\
\hline \multicolumn{2}{|l|}{ CRRT cause } \\
\hline Volume overload, n (\%) & $157(13.92 \%)$ \\
\hline Metabolic acidosis, n (\%) & $242(21.45 \%)$ \\
\hline Hyperkalemia, n (\%) & $57(5.05 \%)$ \\
\hline Uremia, n (\%) & $113(10.02 \%)$ \\
\hline Oliguria, n (\%) & $288(25.53 \%)$ \\
\hline Other, n (\%) & $271(24.02 \%)$ \\
\hline \multicolumn{2}{|l|}{ AKI cause } \\
\hline Sepsis, n (\%) & $783(69.41 \%)$ \\
\hline Others, n (\%) & $345(30.59 \%)$ \\
\hline SOFA score & $12.12 \pm 3.55$ \\
\hline APACHE II score & $27.30 \pm 7.98$ \\
\hline
\end{tabular}




\begin{tabular}{|c|c|c|}
\hline Exposure & $\begin{array}{c}\text { 28-days mortality } \\
\text { (OR value, } P \text { ) }\end{array}$ & $\begin{array}{c}\text { 90-days mortality } \\
\text { (OR value, P) }\end{array}$ \\
\hline Age & $1.00(1.00,1.01), 0.28$ & $1.00(1.00,1.01), 0.37$ \\
\hline \multicolumn{3}{|l|}{ Sex } \\
\hline Man & 1 & 1 \\
\hline Female & $0.92(0.72,1.17), 0.49$ & $0.95(0.73,1.23), 0.68$ \\
\hline BMI & $0.97(0.94,0.99), 0.01$ & $0.96(0.94,0.99), 0.01$ \\
\hline MAP & $0.98(0.97,0.98),<0.001$ & $0.97(0.97,0.98),<0.001$ \\
\hline Myocardial infarction & $0.94(0.63,1.40), 0.76$ & $0.81(0.53,1.24), 0.33$ \\
\hline Congestive heart failure & $0.74(0.54,1.01), 0.06$ & $0.84(0.60,1.19), 0.33$ \\
\hline Cerebrovascular disease & $0.82(0.56,1.22), 0.33$ & $0.80(0.53,1.21), 0.29$ \\
\hline peripheral vascular disease & $0.86(0.47,1.57), 0.63$ & $0.81(0.43,1.51), 0.50$ \\
\hline Dementia & $0.60(0.32,1.11), 0.10$ & $0.57(0.30,1.06), 0.08$ \\
\hline Diabetes mellitus & $0.80(0.62,1.02), 0.08$ & $0.71(0.54,0.92), 0.01$ \\
\hline Hypertension & $0.57(0.45,0.73),<0.001$ & $0.55(0.42,0.71),<0.001$ \\
\hline COPD & $0.69(0.44,1.09), 0.11$ & $0.67(0.42,1.08), 0.10$ \\
\hline Mechanical ventilation & $2.41(1.81,3.21),<0.001$ & $2.39(1.78,3.22),<0.001$ \\
\hline $\mathrm{K}^{+}$ & $1.00(0.90,1.12), 0.99$ & $1.03(0.92,1.16), 0.59$ \\
\hline $\mathrm{HCO}^{-}$ & $0.98(0.96,1.00), 0.09$ & $1.00(0.97,1.02), 0.74$ \\
\hline Phosphate & $1.45(1.33,1.58),<0.001$ & $1.38(1.26,1.51),<0.001$ \\
\hline WBC & $1.00(1.00,1.00), 0.08$ & $1.00(1.00,1.00), 0.05$ \\
\hline $\mathrm{Hb}$ & $0.93(0.88,0.98), 0.01$ & $0.90(0.85,0.95),<0.001$ \\
\hline BUN & $1.00(1.00,1.01), 0.19$ & $1.01(1.00,1.01), 0.002$ \\
\hline $\mathrm{Cr}$ & $0.84(0.78,0.91),<0.001$ & $0.87(0.80,0.94),<0.001$ \\
\hline Alb & $0.49(0.39,0.61),<0.001$ & $0.43(0.34,0.55),<0.001$ \\
\hline CRP & $1.00(1.00,1.00), 0.18$ & $1.00(1.00,1.00), 0.33$ \\
\hline GFR & $1.01(1.00,1.01), 0.01$ & $1.01(1.00,1.02), 0.007$ \\
\hline APACHE II score & $1.05(1.04,1.07),<0.001$ & $1.05(1.04,1.07),<0.001$ \\
\hline SOFA score & $1.26(1.21,1.31),<0.001$ & $1.27(1.22,1.33),<0.001$ \\
\hline \multicolumn{3}{|l|}{ AKIN stage } \\
\hline 2 & 1 & 1 \\
\hline 3 & $0.92(0.70,1.22), 0.57$ & $0.97(0.73,1.31), 0.86$ \\
\hline CCI score & $1.15(1.09,1.22),<0.001$ & $1.16(1.09,1.23),<0.001$ \\
\hline \multicolumn{3}{|l|}{ CRRT cause } \\
\hline Volume overload & 1 & 1 \\
\hline Metabolic acidosis & $1.78(1.17,2.69), 0.007$ & $1.78(1.14,2.78), 0.01$ \\
\hline Hyperkalemia & $1.77(0.94,3.35), 0.08$ & $2.01(0.98,4.10), 0.06$ \\
\hline Uremia & $1.04(0.64,1.68), 0.88$ & $1.10(0.66,1.84), 0.70$ \\
\hline Oliguria & $1.06(0.72,1.57), 0.76$ & $1.13(0.75,1.70), 0.55$ \\
\hline Others & $1.54(1.03,2.29), 0.04$ & $1.51(0.99,2.30), 0.06$ \\
\hline \multicolumn{3}{|l|}{ AKI cause } \\
\hline Non-sepsis & 1 & 1 \\
\hline Sepsis & $1.05(0.81,1.36), 0.72$ & $1.28(0.97,1.69), 0.08$ \\
\hline
\end{tabular}


Table3. multi-factor regression analysis

\begin{tabular}{lll}
\hline Exposure & Non-adjusted OR, P value & Adjusted OR, P value \\
\hline 28-days mortality & & \\
\hline SOFA score & $1.25(1.20,1.31),<0.001$ & $1.38(1.27,1.51),<0.001$ \\
APACHE II score & $1.05(1.04,1.07),<0.001$ & $1.04(1.01,1.07), 0.004$ \\
\hline 90-days mortality & & $1.40(1.28,1.52),<0.001$ \\
\hline SOFA score & $1.27(1.22,1.33),<0.001$ & $1.04(1.01,1.07), 0.01$ \\
APACHE II score & $1.054(1.036,1.072),<0.001$ & \\
\hline
\end{tabular}

Adjusted variables: Age; Sex; BMI; Myocardial infarction; Congestive heart failure; Cerebrovascular disease; peripheral vascular disease; Diabetes mellitus; Dementia; Hypertension; COPD; CRRT cause; CCI; Alb; CRP; GFR; Cr; Mechanical ventilation; WBC; Hb; BUN; K+; HCO3; AKI cause; Phosphate; AKIN (acute kidney injury network stages); Mechanical ventilation; CRRT dose.

Table4. multivariate subgroup analysis in AKI cause

\begin{tabular}{lcc}
\hline 28-days mortality & Non-adjusted OR, P value & Adjusted OR, P value \\
\hline Sepsis & $1.24(1.18,1.30),<0.001$ & $1.38(1.23,1.55),<0.001$ \\
\hline SOFA score & $1.05(1.03,1.07),<0.001$ & $1.03(0.99,1.07), 0.12$ \\
APACHE II score & $1.29(1.19,1.39),<0.001$ \\
\hline Non-sepsis & $1.07(1.04,1.10),<0.001$ & $1.66(1.26,2.19),<0.001$ \\
\hline SOFA Score & Non-adjusted OR, P value & $1.04(0.97,1.12), 0.23$ \\
APACHE II score & $1.26(1.19,1.32),<0.001$ & Adjusted OR, P value \\
\hline 90-days mortality & $1.04,(1.02,1.07),<0.001$ & $1.41(1.26,1.58),<0.001$ \\
\hline Sepsis & & $1.02(0.98,1.06), 0.31$ \\
\hline SOFA score & $1.31(1.21,1.42),<0.001$ \\
APACHE II score & $1.08(1.04,1.11),<0.001$ & $1.36(1.06,1.74), 0.02$ \\
\hline Non-sepsis & $1.03(0.95,1.12), 0.52$ \\
\hline SOFA Score & & \\
APACHE II score & & \\
\hline
\end{tabular}

Adjusted variables: Age; Sex; BMI; Myocardial infarction; Congestive heart failure; Cerebrovascular disease; peripheral vascular disease; Diabetes mellitus; Dementia; Hypertension; COPD; CRRT cause; CCI; Alb; CRP; GFR; Cr; Mechanical ventilation; WBC; Hb; BUN; K+; HCO3-; Phosphate; AKIN (acute kidney injury network stages); Mechanical ventilation; CRRT dose.

\section{Figures}




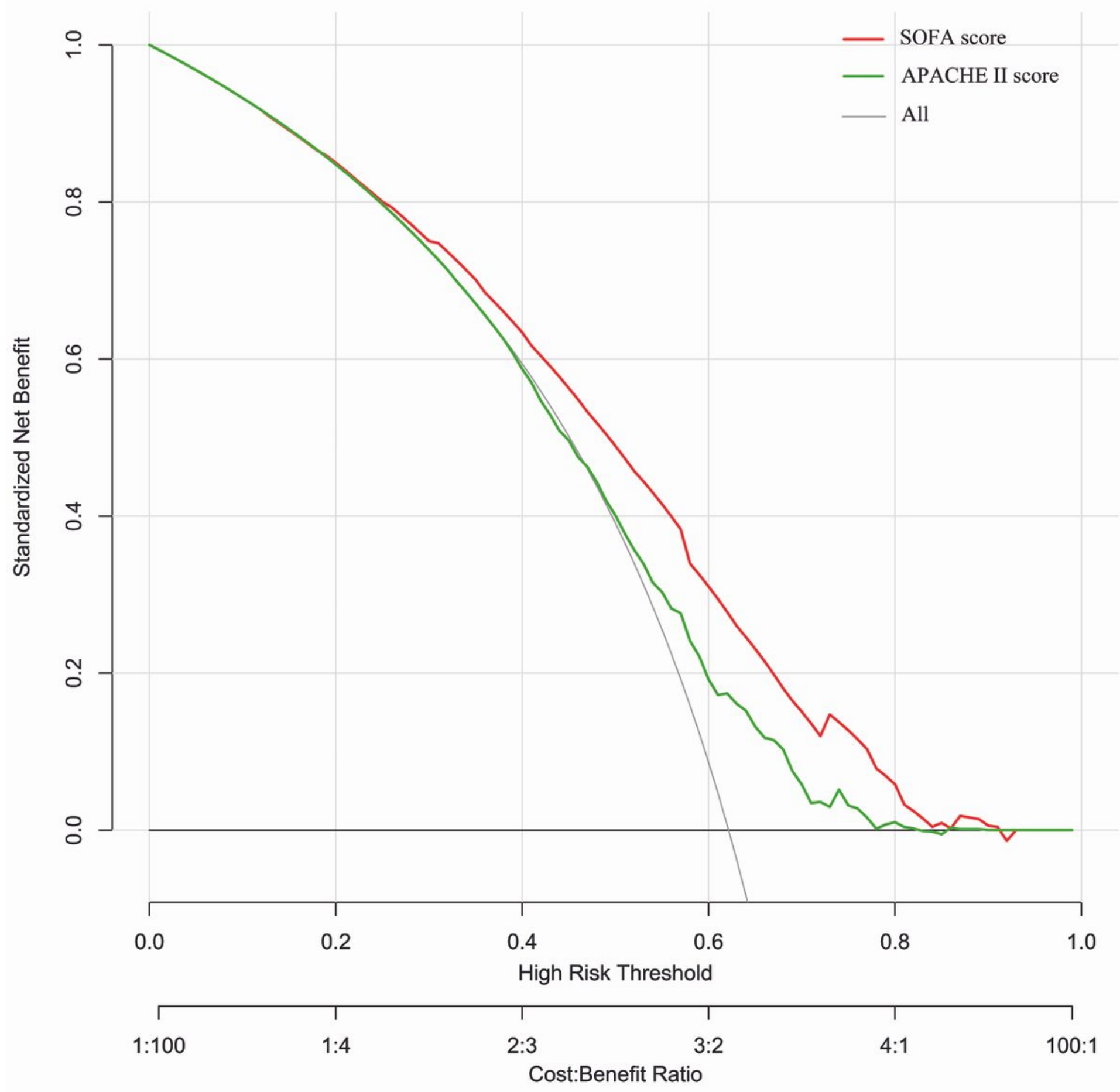

Figure 1

Decision curve analysis for 28-days mortality 
ROC curve for 28-days mortality

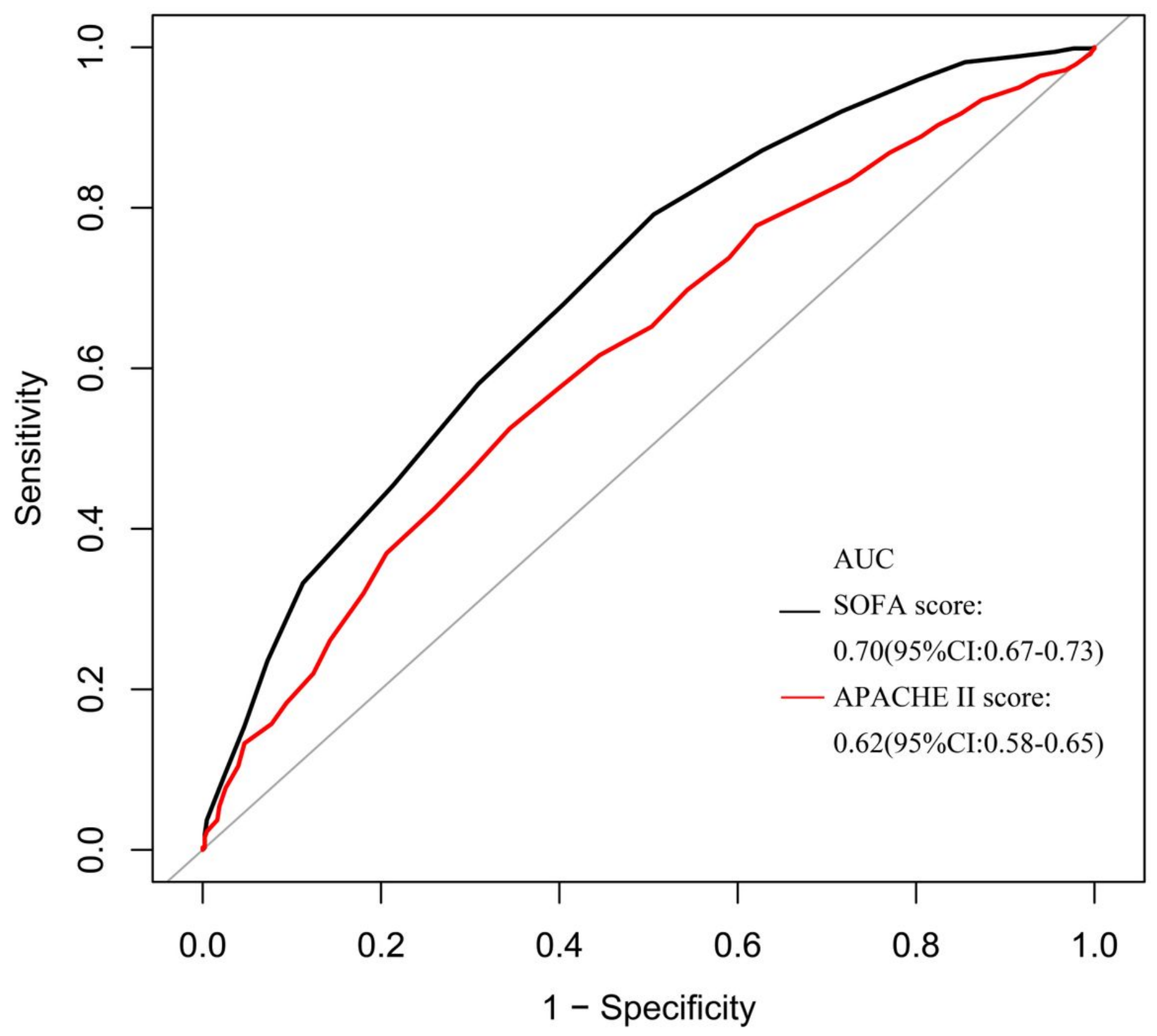

Figure 2

ROC curve for 28-days mortality 


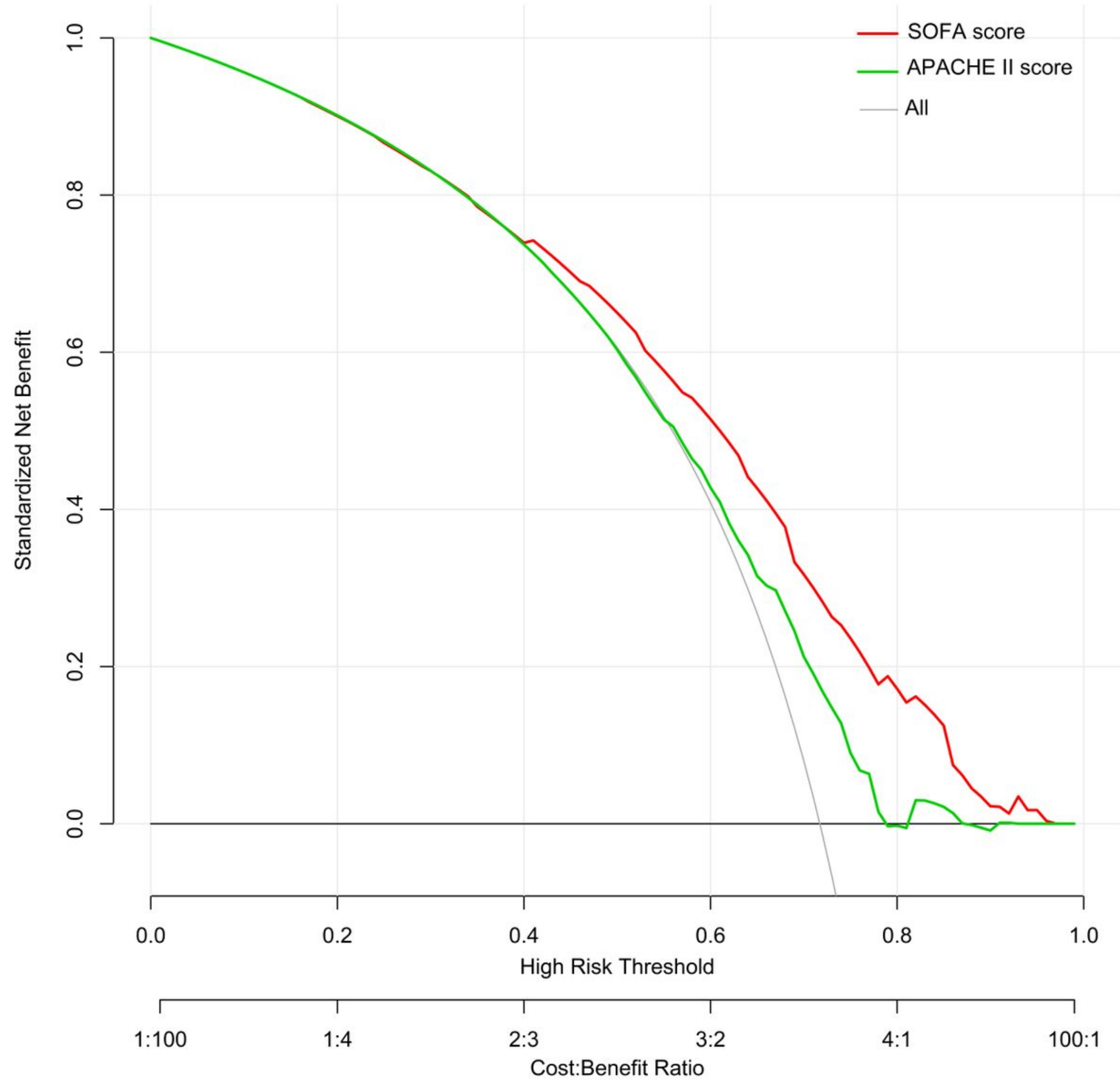

Figure 3

Decision curve analysis for 90 -days mortality 
ROC curve for 90-days mortality

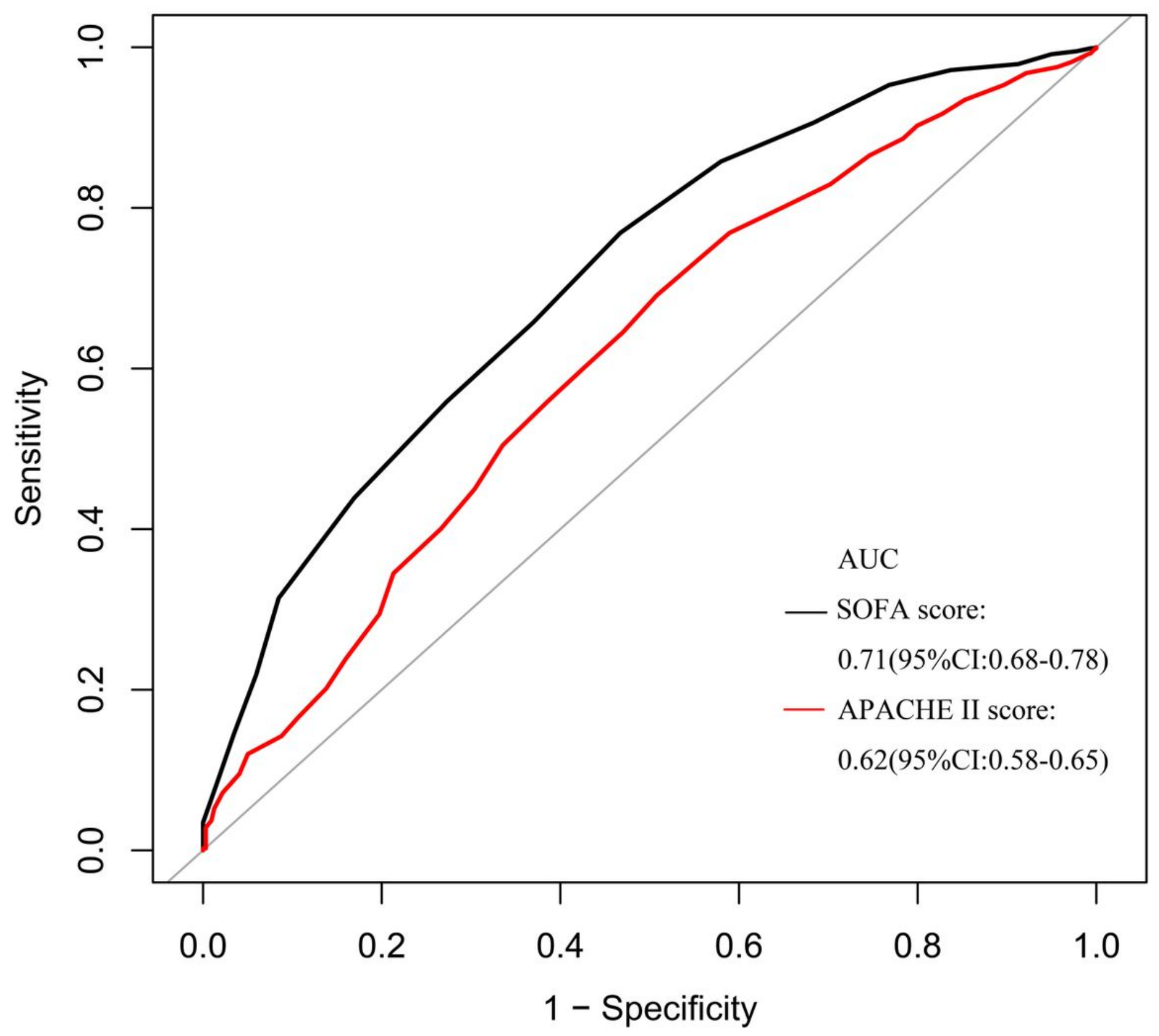

Figure 4

ROC curve for 90 -days mortality 